\title{
Vitamin D - what is normal according to latest research and how should we deal with it?
}

\author{
Author: Neil JL Gittoes ${ }^{\mathrm{A}}$
}

Vitamin D deficiency is a public health concern. Mediated by classical endocrine effects, vitamin $D$ deficiency is causally linked with bone and calcium disorders. Non-endocrine actions of vitamin $D$ are also widely recognised and these effects are mediated by local tissue activation of vitamin $D$ bringing about intracrine effects in non-classical sites. Supported by large volumes of observational studies linking low circulating vitamin D with negative outcomes for many common disease states, there is growing interest that vitamin $D$ may be central to the pathology and outcomes of many common diseases, including cardiovascular, cancer and autoimmune conditions. This article explores the quality of evidence linking vitamin $D$ and various disease outcomes, and furthermore describes some of the cellular and molecular mechanisms of vitamin D action that may help explain some of the incongruity of data observed in observational versus interventional studies of vitamin D supplementation.

KEYWORDS: Vitamin D, osteomalacia, rickets, vitamin D receptor, cardiovascular mortality, cancer

\section{Introduction}

Vitamin D supports an expanding plethora of references within scientific and clinical journals, as well as multiple general media reports proclaiming associations between low levels of circulating vitamin D and multiple health outcomes. Vitamin $\mathrm{D}$ is a steroid hormone precursor synthesised within skin under the action of ultraviolet B radiation (sunlight). Dietary availability of vitamin D is generally limited, although oily fish provides a good source.

From a clinical standpoint, vitamin D deficiency has historically been most widely recognised in the context of endocrine dysfunction, with disturbed calcium and bone homeostasis causally linked to rickets in children and osteomalacia in adults. In more recent times, 'non-classical' actions of vitamin D have been described, mediated by local, tissue-specific activation of vitamin D allowing intracrine

Author: ${ }^{\text {A }}$ consultant and honorary senior lecturer, Department of Endocrinology, University Hospitals Birmingham and University of Birmingham, Birmingham, UK functions of vitamin D separate to its established endocrine role in calcium/bone homeostasis. It is via the former action that vitamin $\mathrm{D}$ has been considered as a potentially important factor in health maintenance and in many (common) disease states. Recognition of widespread expression of vitamin $\mathrm{D}$ receptors beyond tissues involved in classical endocrine pathways adds validity to the assertion that vitamin $\mathrm{D}$ has more pleiotropic effects than first considered. Additionally, the enzyme ( $1 \alpha$-hydroxylase) responsible for conversion of inactive 25-hydroxyvitamin D to its biologically active form, 1,25-dihydroxyvitamin $\mathrm{D}$, is not exclusively expressed in the kidneys but is also present in other extra-renal tissues, further adding credibility to the non-endocrine/non-classical actions of vitamin D.

\section{Vitamin D beyond calcium and bone}

Numerous observational studies have demonstrated strong associations between low circulating concentrations of 25-hydroxyvitamin D and many non-skeletal diseases (eg cardiovascular disease, certain cancers and autoimmune diseases) and their outcomes. Recent meta-analyses have highlighted these strong inverse correlations between low vitamin D concentrations and multiple health outcomes including cause-specific and all-cause mortality. ${ }^{1-3}$ These same meta-analyses examined (the relatively small number of) randomised controlled trials of supplementation, exploring potential causal associations with vitamin $\mathrm{D}$. Two studies showed small reductions in all-cause mortality in older individuals (5 and 11\%). ${ }^{1,3}$ Theodoratou and colleagues ${ }^{2}$ showed a beneficial effect of vitamin D supplementation on birth weight and reduced dental caries. Cause-specific mortality was not influenced by supplementation in any of the meta-analyses.

The broad themes and conclusions drawn from these largescale meta-analyses were that more research was required and that a causal link between vitamin D status and health outcomes cannot be inferred. Reverse causality must also be considered as an explanation for observed associations as there is a compelling case that 'ill health' in its own right often results in reduced UV exposure and suboptimal nutritional status, among other factors, which may lower circulating vitamin D. Additional confounding factors that make it difficult to derive definitive conclusions from vitamin $\mathrm{D}$ treatment studies include supplementation of populations irrespective of risk factors for vitamin D deficiency/insufficiency. Any potential 
beneficial effect of supplementation may be envisaged to be more apparent in those with quantitatively more significant reductions in vitamin D. Moreover, there is little consensus around the target concentration of 25-hydroxyvitamin D that may have beneficial effects on non-classical disease associations, and only in a minority of studies has total vitamin $\mathrm{D}$ been measured at baseline and following supplementation to demonstrate effective reversal of any deficiency.

Due to numerous limitations, some identified above, it is currently very difficult to provide conclusive evidence regarding causality or not using the current evidence base of supplementation studies. Alternative approaches using Mendelian randomisation have been adopted in an attempt to reduce confounding and glean further insight into low circulating vitamin D and multiple health outcomes. Afzal and colleagues ${ }^{4}$ looked at genetic variants of two genes encoding key enzyme regulators of endogenous production of 25-hydroxyvitmimn $\mathrm{D}$, dehydrocholesterol reductase (DHCR7) and 25 hydroxylase (CYP2R1) in a subgroup of 30,000 within a Danish cohort of $95,000 .{ }^{4}$ Polymorphic variants in these two genes result in differential efficiencies in synthesising 25 -hydroxyvitamin $\mathrm{D}$; thus by examining allele scores in these variants it was possible to determine those individuals who have endogenously (genetically determined) lower vitamin D, and any interaction this may have with a broad array of health outcomes. Using the approach outlined, the group modelled the effect of a genetically determined $20 \mathrm{nmol} / \mathrm{L}$ reduction in circulating 25-hydroxyvitamin D and its impact on all-cause and cause-specific mortality. The study demonstrated increased all-cause mortality (odds ratio (OR) 1.3 (95\% confidence interval (CI) 1.06-1.61)), cancer mortality (OR 1.43 (95\% CI 1.02-1.99)) and other-cause mortality (OR 1.44 (95\% CI 1.01-2.04)) but there was no increased cardiovascular mortality in those with lower vitamin D. This Mendelian randomisation study adds some weight to a possible causal association between intrinsically low vitamin D and health outcomes; however such large-scale genetic studies do not provide a direct surrogate for the gold standard of randomised controlled trials.

\section{What does abrogated function of the vitamin D receptor tell us?}

Mouse models of vitamin D receptor (VDR) ablation have abrogated vitamin D signalling with severe osteomalacia, but careful phenotyping reveals no increased risk of malignancies, immune deficits or cardiovascular pathology. ${ }^{5}$ VDR knockout models do not develop spontaneous tumours although they do have an increased susceptibility to carcinogen-induced tumours of the skin and breast. ${ }^{6,7} \mathrm{An}$ increased risk of inflammatory bowel disease and reduced interferon- $\gamma$ has also been shown. ${ }^{8}$ Observed T-cell and macrophage dysfunction is secondary to associated hypocalcaemia as this immune dysfunction resolves with correction of serum calcium. ${ }^{9}$ VDR knockout mice have higher blood pressure mediated via an activated renin-angiotensin system. ${ }^{10}$

The very rare human disease of hereditary vitamin D dependent rickets is mediated by mutations in VDR rendering it ineffective. There is no apparent early excess risk of cancers nor autoimmune conditions or hypertension in these patients. ${ }^{11}$ Due to rarity, the numbers of patients studied is small so no firm conclusions can be drawn from the human disease state.
Taken together, however, observations from murine and human functional vitamin D deficiency states (via VDR ablation) do not clearly support a causal link between vitamin D, increased cancer risk, autoimmunity or significant cardiovascular pathology.

\section{Vitamin D physiology}

Vitamin D physiology is well characterised with respect to its endocrine action. 25-hydroxylated vitamin $\mathrm{D}$ is activated within the kidney to 1,25-dihydroxyvitamin D under the action of $1 \alpha$-hydroxylase. 1,25 -dihydroxyvitamin $\mathrm{D}$ is the active form of vitamin $\mathrm{D}$, capable of biological effects. The active form circulates free or bound (discussed later) in the systemic circulation to target cells to achieve its endocrine effects. More recently, extra-renal activation of vitamin $\mathrm{D}$ by $1 \alpha$-hydroxylase has been reported at sites other than the kidneys, ${ }^{12}$ including cancer and immune cells, which has relevance to many disease states. Local tissue-specific production of 1,25-dihydroxyvitamin $\mathrm{D}$ allows intracrine actions.

In vitro studies provide important insight into local effects of 1,25-dihydroxyvitamin D. In cancer cells there is broadly reduced proliferation, invasion, angiogenesis and tendency to metastasise with corresponding enhanced apoptosis and differentiation. ${ }^{13}$ In immune cells, vitamin $\mathrm{D}$ reduces inflammation, dendritic cell maturation, cytokine production and antigen presentation, while leading to increased monocyte differentiation, improved bacterial killing and enhanced suppressor T-cell function. ${ }^{14}$ Overall, the in vitro effects of 1,25-dihydroxyvitamin are 'anti-cancer' and 'antiinflammatory', which from a mechanistic standpoint support important putative roles of vitamin D in these disease states.

\section{Vitamin D and the free hormone hypothesis}

Vitamin D is highly lipophilic and relies on serum carrier proteins to ensure effective delivery to target cells. The inactive form of vitamin D is managed differently in different cell types and tissue-specific expression of $1 \alpha$-hydroxylase determines activation to 1,25-dihydroxyvitamin D. Conventional laboratory assays for vitamin D measure total (25-hydroxy) vitamin $\mathrm{D}$, which incorporates vitamin $\mathrm{D}$ bound to vitamin D binding protein (DBP, 90\%), albumin (9.9\%) and a very small fraction of free vitamin $\mathrm{D}$. The ability of different tissues to handle the circulating forms of vitamin $\mathrm{D}$ is very different. The kidney has appropriate intracellular machinery (principally megalin) ${ }^{15}$ to handle vitamin D bound to DBP, whereas non-renal tissues are generally less able to deal with this fraction of vitamin D. Some cancer cell lines are capable of this function. ${ }^{16,17}$ In general, non-renal tissues are more reliant on the bioavailable pool of vitamin $\mathrm{D}$, which incorporates albumin-bound and free vitamin D that constitutes only $0.1 \%$ of the total vitamin D pool. 25-hydroxyvitamin D binds to DBP with much higher affinity than 1,25-dihydroxyvitamin $\mathrm{D}$, and thus it is likely that DBP has a much greater impact on 25-hydroxyvitamin D-mediated intracrine responses. In this way, quantitative and qualitative factors in DBP may be important in determining the impact of vitamin $\mathrm{D}$ on various (non-classical) health parameters.

Polymorphisms in DBP account for different abundances of binding protein and different affinities for binding vitamin D. 
Powe and colleagues ${ }^{18}$ studied black and white Americans and characterised DBP genotypes and measured concentrations of circulating DBP to determine whether they differed between races, possibly accounting for observed racial differences in manifestations of vitamin D deficiency. Although black Americans had lower total vitamin D than whites, they had higher bone mineral densities, higher serum calcium and only marginally higher parathyroid hormone concentrations. When polymorphic variants of DBP were taken into account, the estimated bioavailable vitamin $\mathrm{D}$ was very similar between black and white Americans. The authors postulated that lower levels of DBP in black Americans may provide protection against clinical manifestations of endocrine parameters of vitamin $\mathrm{D}$ deficiency despite low levels of circulating total 25 -hydroxyvitamin D. It remains to be determined what direct impact quantitative and qualitative changes in DBP may have on non-classical vitamin D actions (predominantly mediated via intracrine mechanisms). As it is unbound 25-hydroxyvitamin D that drives many of the non-classical actions, it is likely that DBP is an important regulator and determinant of intracrine functions. Bioavailable vitamin D is therefore likely to be a better marker of clinically relevant vitamin $\mathrm{D}$ action rather than total vitamin $\mathrm{D}$.

\section{What target levels of total vitamin D should we aim for?}

Non-classical effects of vitamin D are likely to be mediated via distinct intracrine actions rather than systemic endocrine effects. It is quite feasible therefore that target concentrations of total vitamin D may be different to achieve a desired endocrine effect compared to putative intracrine effects on immune function and cancer prevention for instance.

There is some consensus regarding optimal vitamin D levels to prevent endocrine disease. However, due to lack of consistency around causality, there is no consensus as to what concentration of circulating vitamin D may be optimal to 'prevent' deleterious consequences in non-classical vitamin D pathways. Currently vitamin D supplementation is not advocated to prevent chronic diseases. ${ }^{19}$ The following sections therefore are restricted to vitamin $\mathrm{D}$ treatment for prevention of endocrine disease in the form of musculoskeletal disease and disturbance of mineral ion homeostasis.

There is consensus between the Institute of Medicine guideline $e^{20}$ and the National Osteoporosis Society guideline $e^{21}$ that a sufficient concentration of total vitamin $\mathrm{D}$ to achieve appropriate bone health is $50 \mathrm{nmol} / \mathrm{L}$ and that deficiency is defined as less than $30 \mathrm{nmol} / \mathrm{L}$. The American Endocrine Society ${ }^{22}$ derived different thresholds, recognising sufficiency at greater than $75 \mathrm{nmol} / \mathrm{L}$ and deficiency less than $50 \mathrm{nmol} / \mathrm{L}$. The National Osteoporosis Society guideline advised treatment of circulating total vitamin $\mathrm{D}$ between $30-50 \mathrm{nmol} / \mathrm{L}$ in the presence of additional clinical risk factors. ${ }^{21}$

\section{Who should we test and how should we treat vitamin D deficiency?}

A targeted, rather than population-based screening approach to vitamin $\mathrm{D}$ testing is advocated. Patients with diseases with outcomes that may be improved with vitamin $\mathrm{D}$ supplementation (eg osteomalacia and osteoporosis) may be tested as well as patients with symptoms that could be attributed to vitamin D deficiency (eg suspected osteomalacia and widespread pain). There is no absolute requirement for vitamin $\mathrm{D}$ testing in individuals who are asymptomatic but who are at increased risk of vitamin D deficiency; empirical supplementation of at risk groups should be considered. ${ }^{23}$

Effective treatment of vitamin D deficiency can take many practical forms but there are some key aims for treatment. Adequate doses should be used to ensure correction of deficiency, clinical consequences of vitamin D deficiency should be reversed in a timely manner and maintenance doses administered to avoid relapse while avoiding toxicity. Vitamin $\mathrm{D}_{3}$ is the preferred form for supplementation as vitamin $\mathrm{D}_{2}$ is cleared more quickly ${ }^{24}$ and has lower tissue bioavailability. ${ }^{25}$ Fixed loading doses of high-strength vitamin D (total dose of approximately 300,000 IU in divided doses) should be considered in those with severe symptoms of deficiency and in those requiring treatment with potent antiresorptive drugs such as intravenous zoledronic acid or subcutaneous denosumab. After loading, a maintenance dose (typically 800-2,000 IU/day) of vitamin D should be prescribed to avoid further deficiency. A maintenance dose strategy (without requirement for loading) can be considered in those patients with deficiency where timeliness of reversal of vitamin D deficiency is less pressing. Routine measurements of serum total vitamin D are not required in patients receiving supplementation. ${ }^{21}$

\section{Conclusions}

Vitamin D deficiency remains a public health concern and it is accepted that in those with underlying bone diseases, achieving satisfactory levels throughout the year is important to optimise musculoskeletal outcomes. The burgeoning observational data linking low vitamin $\mathrm{D}$ with multiple chronic diseases and outcomes is intriguing yet there is a paucity of reliable, appropriately powered randomised controlled trials to help determine potential causality. There remain concerns around potential reverse causality and multiple confounders that mean that we should not over extrapolate clinically to recommend blanket supplementation to prevent non-classical diseases. For instance, more novel approaches to minimise confounders using Mendelian randomisation have provided further clues that support potential causality.

Experiments of nature and murine models of abrogated vitamin $\mathrm{D}$ function reveal no clear propensity for non-classical vitamin $\mathrm{D}$-associated diseases. Insights into local tissue-specific activation of vitamin D to mediate intracrine effects in extrarenal tissues, and the potential centrality of DBP in determining free hormone concentrations, provides a potential mechanism whereby vitamin D may mediate its non-classical actions.

There are many unknowns embedded within vitamin D metabolism and action and there remains controversy surrounding even the basic parameter of what constitutes a satisfactory circulating concentration of vitamin D. It is feasible that what constitutes 'satisfactory' for endocrine/ musculoskeletal function may be somewhat different to a 'satisfactory' level to mediate putative non-classical actions. Clinical trials are underway that attempt to unlock the issue of vitamin D supplementation and potential prevention of chronic, non-classical diseases, and these are due to start reporting in 2017 . 


\section{References}

1 Chowdhury R, Kunutsor S, Vitezova A et al. Vitamin D and risk of cause specific death: systematic review and meta-analysis of observational cohort and randomised intervention studies. $B M J$ 2014;348:g1903.

2 Theodoratou E, Tzoulaki I, Zgaga L, Ioannidis JP. Vitamin D and multiple health outcomes: umbrella review of systematic reviews and meta-analyses of observational studies and randomised trials. BMJ 2014;348:g2035.

3 Autier P, Boniol M, Pizot C, Mullie P. Vitamin D status and ill health: a systematic review. Lancet Diabetes Endocrinol 2014;2:76-89.

4 Afzal S, Brondum-Jacobsen P, Bojesen SE, Nordestgaard BG. Genetically low vitamin D concentrations and increased mortality: Mendelian randomisation analysis in three large cohorts. BMJ 2014;349:g6330.

5 Demay MB. Physiological insights from the vitamin D receptor knockout mouse. Calcif Tissue Int 2013;92:99-105.

6 Zinser GM, Welsh J. Vitamin D receptor status alters mammary gland morphology and tumorigenesis in MMTV-neu mice. Carcinogenesis 2004;25:2361-72.

7 Zinser GM, Sundberg JP, Welsh J. Vitamin D(3) receptor ablation sensitizes skin to chemically induced tumorigenesis. Carcinogenesis 2002;23:2103-9.

8 Froicu M, Weaver V, Wynn TA et al. A crucial role for the vitamin $\mathrm{D}$ receptor in experimental inflammatory bowel diseases. Mol Endocrinol 2003;17:2386-92.

9 Mathieu C, Van Etten E, Gysemans C et al. In vitro and in vivo analysis of the immune system of vitamin D receptor knockout mice. J Bone Miner Res 2001;16:2057-65.

10 Li YC, Kong J, Wei M et al. 1,25-Dihydroxyvitamin D(3) is a negative endocrine regulator of the renin-angiotensin system. J Clin Invest 2002;110:229-38.

11 Feldman D, P JM. Mutations in the vitamin D receptor and hereditary vitamin D-resistant rickets. Bonekey Rep 2014;3:510.

12 Hewison M, Burke F, Evans KN et al. Extra-renal 25-hydroxyvitamin D3-1alpha-hydroxylase in human health and disease. J Steroid Biochem Mol Biol 2007;103:316-21.

13 Feldman D, Krishnan AV, Swami S et al. The role of vitamin D in reducing cancer risk and progression. Nat Rev Cancer 2014;14: $342-57$.
14 Chun RF, Peercy BE, Orwoll ES et al. Vitamin D and DBP: the free hormone hypothesis revisited. J Steroid Biochem Mol Biol 2014;144 Pt A:132-7.

15 Nykjaer A, Dragun D, Walther D et al. An endocytic pathway essential for renal uptake and activation of the steroid $25-(\mathrm{OH})$ vitamin D3. Cell 1999;96:507-15.

16 Rowling MJ, Kemmis CM, Taffany DA, Welsh J. Megalin-mediated endocytosis of vitamin $\mathrm{D}$ binding protein correlates with 25-hydroxycholecalciferol actions in human mammary cells. J Nutr 2006;136:2754-9.

17 Chlon TM, Taffany DA, Welsh J, Rowling MJ. Retinoids modulate expression of the endocytic partners megalin, cubilin, and disabled-2 and uptake of vitamin D-binding protein in human mammary cells. J Nutr 2008;138:1323-8.

18 Powe CE, Karumanchi SA, Thadhani R. Vitamin D-binding protein and vitamin D in blacks and whites. N Engl J Med 2014;370:880-1.

19 Meyer HE, Holvik K, Lips P. Should vitamin D supplements be recommended to prevent chronic diseases? BMJ 2015;350:h321.

20 Institute of Medicine. Dietary reference intakes for calcium and vitamin D. Washington DC: IoM, 2011.

21 Aspray TJ, Bowring C, Fraser W et al. National osteoporosis society vitamin D guideline summary. Age Ageing 2014;43:592-5.

22 Holick MF, Binkley NC, Bischoff-Ferrari HA et al. Evaluation, treatment, and prevention of vitamin D deficiency: an Endocrine Society clinical practice guideline. J Clin Endocrinol Metab 2011;96:1911-30.

23 UK CMO. Vitamin D - Advice on supplements for at risk groups. London: DoH, 2012.

24 Romagnoli E, Mascia ML, Cipriani C et al. Short and long-term variations in serum calciotropic hormones after a single very large dose of ergocalciferol (vitamin D2) or cholecalciferol (vitamin D3) in the elderly. J Clin Endocrinol Metab 2008;93:3015-20.

25 Heaney RP, Recker RR, Grote J et al. Vitamin D(3) is more potent than vitamin D(2) in humans. J Clin Endocrinol Metab 2011;96:E447-52.

Address for correspondence: Prof NJL Gittoes, University Hospitals Birmingham and University of Birmingham, Edgbaston, Birmingham B15 2TH, UK. Email: neil.gittoes@uhb.nhs.uk 